\title{
Energy and orientation dependence of neutron depolarization in a large single crystal of ferromagnetic holmium
}

\author{
V. P. Alfimenkov, A. N. Chernikov, L. Lason, ${ }^{\text {a) }}$ Yu. D. Mareev, V. V. Novitsky, \\ L. B. Pikelner, V. R. Skoy, and M. I. Tsulaya \\ Frank Laboratory of Neutron Physics, Joint Institute for Nuclear Research, 141980 Dubna, \\ Russian Federation \\ C. R. Gould and D. G. Haase ${ }^{\text {b) }}$ \\ Physics Department, North Carolina State University, Raleigh, North Carolina 27695-8202 and the \\ Triangle Universities Nuclear Laboratory, Durham, North Carolina 27708-0308 \\ N. R. Roberson \\ Physics Department, Duke University, Durham, North Carolina 27708-0305 and the Triangle Universities \\ Nuclear Laboratory, Durham, North Carolina 27708-0308
}

(Received 23 February 1996; accepted for publication 25 October 1996)

\begin{abstract}
We report measurements of the depolarization of epithermal neutrons $(1.7-59 \mathrm{eV})$ in magnetic domains of a $2.0-\mathrm{cm}$-diam cylindrical single crystal of ferromagnetic holmium. The neutrons were polarized by a dynamically polarized proton target and polarization analyzed using a statically polarized rare-earth spin filter. Based on the dependence of the depolarization on neutron energy and crystal orientation, we determined the domains to be laminar, or needlelike, with the long axis slightly deflected from the $c$ crystalline axis and having an average width of $59 \mu \mathrm{m}$. (C) 1997 American Institute of Physics. [S0021-8979(97)04303-X]
\end{abstract}

\section{INTRODUCTION}

The measurement of the depolarization of beams of polarized thermal neutrons in materials has been used to determine statistical measures of magnetic domain sizes and orientations. ${ }^{1}$ Such measurements are usually limited to thin samples because low energy neutrons are rapidly depolarized in strong magnetic fields in domains. In the present work we investigate domains in a thick single crystal of ferromagnetic holmium by their depolarizing effects on polarized neutrons of 1.7-59 eV energies. We have determined the dependence of the depolarization on the orientation of the crystal and the energy of the neutrons. We show how a simple model can be used to deduce the geometry and average size of the magnetic domains.

The helical ferromagnetic phase of holmium exists below $20 \mathrm{~K}$. The $c$ hexagonal crystalline axis is the screw axis and the electronic moments have a turn angle of $30^{\circ}$ per atomic layer. There is a net magnetic moment of $1.7 \mathrm{Bohr}$ magnetons per unit cell produced by the canting of the electronic moments $10^{\circ}$ out of the $a-b$ plane. The saturation internal field in the $c$ direction is $0.664 \mathrm{~T}$ and in the $a-b$ plane $3.87 \mathrm{~T}^{2}$

The polarization $f_{n o}$ of a beam of neutrons traversing a ferromagnetic material is reduced to a value $f_{n}$ by the precession of the neutron magnetic moments in the local fields of the individual domains. The precession $\Delta \Theta$ of a neutron of energy $E$ in passing through a holmium domain of length $\delta$ is given by

$$
\Delta \Theta=\left(\gamma_{n}\right) B_{\perp} \frac{\delta}{\sqrt{\left(\frac{2 E}{m_{n}}\right)}},
$$

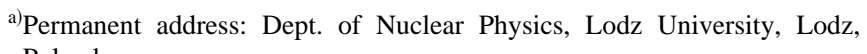
Poland.

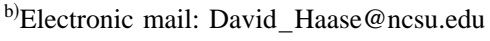

where $\gamma_{n}$ is the neutron gyromagnetic ratio, $m_{n}$ is the mass of the neutron, and $B_{\perp}$ is the component of the local field perpendicular to the neutron polarization axis. For a holmium domain saturated in the $c$ direction a $59 \mathrm{eV}$ neutron would undergo a precession of $1 \mathrm{rad}$ in $880 \mu \mathrm{m}$.

Halpern and Holstein ${ }^{3}$ showed that for a sample having large randomly oriented domains such that the precession in any one domain is large, the polarization transfer $f_{n} / f_{n o}$ is

$$
f_{n} / f_{n o}=\exp \left(-2 D^{\prime} t\right),
$$

where $t$ is the target thickness and $D^{\prime}$ is an energy independent depolarization parameter. In the limit where the precession in any one domain is small the same relation holds except that $D^{\prime}$ is replaced by an energy dependent parameter

$$
D_{\text {random }}^{\prime}=\frac{\gamma_{n}^{2}\left\langle B_{\perp}^{2} \delta^{2}\right\rangle_{\mathrm{avg}} n}{8\left(\frac{E_{n}}{m_{n}}\right)},
$$

where $n$ is the number of domains per unit length. This formalism was successfully applied to results of depolarization measurements in thin foils of polycrystalline holmium. ${ }^{4}$

The TRIPLE collaboration measured the depolarization of $0.73 \mathrm{eV}$ neutrons in a 2.29 -cm-diam cylindrical single crystal of ferromagnetic holmium. ${ }^{5}$ The neutrons were polarized longitudinally, parallel or antiparallel to the neutron momentum. The magnetic orientation axis of the holmium crystal was rotated from $0^{\circ}$ to $90^{\circ}$ relative to the beam direction. In such a case the magnetization directions of the domains are spatially correlated-magnetized parallel or antiparallel to the $c$ crystalline axis. Following earlier work ${ }^{6}$ it was proposed that the polarization transfer in the holmium crystal could be described as

$$
\begin{aligned}
f_{n} / f_{n o}= & {\left[\cos ^{2} \theta+\sin ^{2} \theta \exp \left(-2 D_{\text {bulk }}^{\prime} t\right)\right] } \\
& \times \exp \left(-2 D_{\text {random }}^{\prime} t\right),
\end{aligned}
$$


where $\theta$ is the angle between the neutron spin and the $c$ axis. The term $D_{\text {random }}^{\prime}$ describes depolarization due to random disorder in domain magnetization directions as in Eq. (1). The $D_{\text {bulk }}^{\prime}$ term is a result of deviations in the correlations of the domains in the bulk material:

$$
2 D_{\text {bulk }}^{\prime} t=\left(\frac{\gamma_{n} B_{\text {int }}}{2 \sqrt{2} v_{n}}\right)^{2} \delta t,
$$

$B_{\text {int }}$ being the total internal field in the domain. The cosine and sine terms in Eq. (2) are multiplied by two different exponentials because of the crystal symmetry: the component of the neutron polarization parallel to the magnetic axis of the domains is preferentially conserved. This model also assumes that the neutron precession in any one domain is small. Although the model could not be tested as to the energy dependence of the depolarization constants, the fit to the data indicated a minimum average domain size of $11 \mu \mathrm{m}$.

\section{EXPERIMENT}

We report measurements of the depolarization of epithermal neutrons in a cylindrical single crystal of ferromagnetic holmium over a range of neutron energies and in two geometrical orientations. This extension of the TRIPLE experiment is made possible by the use of a polarized rare-earth neutron polarization analyzer assembled at the Frank Laboratory. ${ }^{7}$ Neutrons from the IBR-30 pulsed source are polarized by passage through a cryogenic dynamically polarized proton target. The neutron polarization is about 0.6 and the neutron flux exiting the target is $300000 E^{-0.9}$ neutrons/ $\mathrm{s} \mathrm{eV}$. The neutrons then proceed through an adiabatic spin flipper and enter a drift tube in which there is a guide field of $0.04 \mathrm{~T}$.

The holmium target is located in a cryostat at $4.2 \mathrm{~K}$ in the neutron guide field. The cylindrical axis of the crystal is oriented perpendicular to the neutron beam and may be rotated by a shaft inserted into the cryostat. Upon exiting the holmium target the neutron beam is guided to a cryogenic statically polarized natural Dy target. The Dy target consisted of a 1.4-mm-thick stack of foils cooled to $40 \mathrm{mK}$ in a $1.5 \mathrm{~T}$ polarizing field parallel to the plane of the target plate. Resonances in Dy at 1.7, 2.7, 14, 16, 18, 36, and $59 \mathrm{eV}$ have spin dependent neutron transmission ${ }^{8}$ and act as analyzers for neutrons with these energies. The neutrons passing through the Dy analyzer are then counted by a ${ }^{3} \mathrm{He}$ ionization detector $30 \mathrm{~m}$ from the neutron source. The neutron energies are determined by the time-of-flight technique. The distance from the IBR source to the proton target is $9 \mathrm{~m}$, to the holmium target $11 \mathrm{~m}$, and to the Dy analyzer $12 \mathrm{~m}$.

The sign of the neutron polarization was changed by rotating the polarized proton target every $5 \mathrm{~min}$. Each oriention of the holmium crystal was measured in 6-9 h. Neutron yields were collected for both parallel $\left(N_{+}\right)$and antiparallel $\left(N_{-}\right)$neutron and dysprosium spin orientations. An average transmission asymmetry $\langle\epsilon\rangle=\left(N_{+}-N_{-}\right) /\left(N_{+}+N_{-}\right)$proportional to $f_{n}$ was determined by integration over the time-offlight channels corresponding to a particular Dy resonance. ${ }^{7}$ The depolarization was also measured with the holmium target at room temperature. Because holmium is paramagnetic

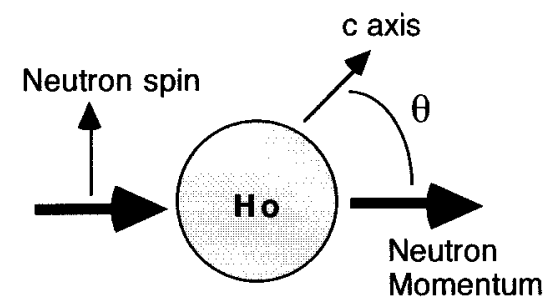

Transverse Geometry

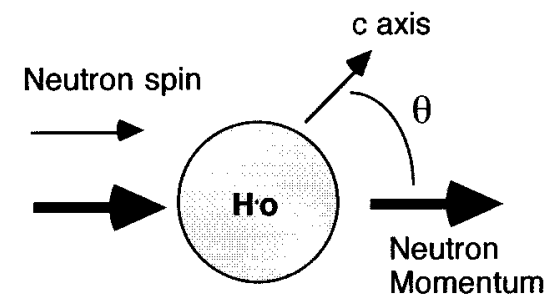

Longitudinal Geometry

FIG. 1. Experimental geometries used for this investigation. In each case the neutron spin, neutron momentum and the $c$ crystalline axis are coplanar. The cylindrical axis of the crystal is perpendicular to that plane. In the transverse geometry the neutron polarization axis is perpendicular to the neutron momentum.

at room temperature, the ratio of the initial polarization $f_{n o}$ to the polarization $f_{n}$ of the transmitted neutron beam was calculated as $f_{n} / f_{n o}=\langle\epsilon\rangle_{4.2 \mathrm{~K}} /\langle\epsilon\rangle_{300 \mathrm{~K}}$.

The holmium cylinder was produced at the Materials Preparation Center at the Ames Laboratory, Iowa State University, and measured $2.0 \mathrm{~cm}$ in diameter and $2.8 \mathrm{~cm}$ in height. The $c$ axis of the crystal was oriented perpendicular to the cylinder axis. This orientation was checked by $\mathrm{x}$-ray and neutron diffraction measurements. Because of the small guide field and the large demagnetization factor of the cylinder, it is assumed that during the measurements the magnetization of the cylinder was negligible. ${ }^{2}$

\section{EXPERIMENTAL RESULTS}

The neutron depolarization was measured in two different arrangements (Fig. 1). In each case the neutron momentum, the neutron spin, and the $c$ axis of the holmium crystal were in the same plane. In the transverse geometry the neutron spin was perpendicular to the neutron momentum, whereas in the longitudinal geometry, the spin was parallel to the neutron momentum.

The polarization transmission $f_{n} / f_{n o}$ was measured in the transverse geometry at ten orientations $\theta=-15^{\circ}-180^{\circ}$. The energy dependence of $f_{n} / f_{n o}$ is shown in Fig. 2. At high energies $f_{n} / f_{n o}$ is proportional to $\exp \left(-\alpha / E_{n}\right)$ as expected and the slope $\alpha$ is dependent upon the orientation of the crystal. The neutron beam was most strongly depolarized when the neutron spin was perpendicular to an axis offset by about $15^{\circ}$ from the holmium $c$ crystalline axis.

The depolarization was also measured in the longitudinal geometry at three orientations of the $c$ axis relative to the beam momentum: $\theta=0^{\circ}, 45^{\circ}$, and $90^{\circ}$. These data (Fig. 3) 


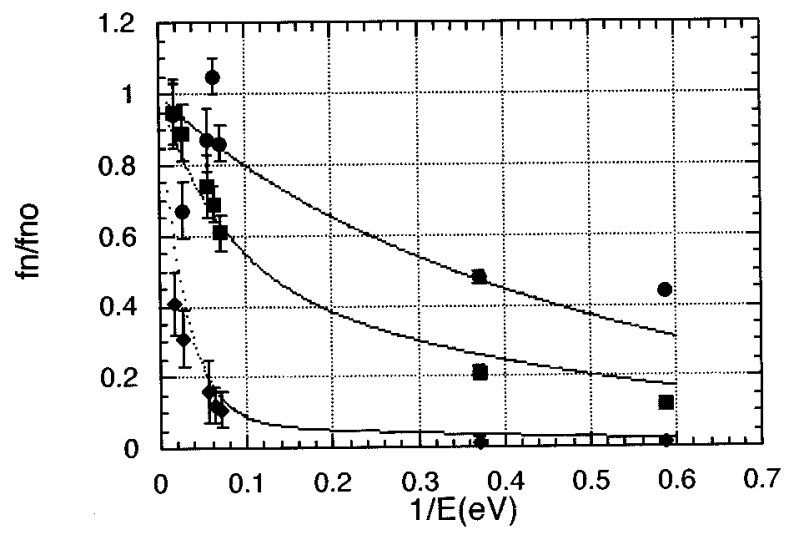

FIG. 2. Energy dependence of the polarization transmission $f_{n} / f_{n o}$ in the transverse geometry, as measured at selected orientation angles. Diamonds, $\theta=0^{\circ}$; squares, $\theta=30^{\circ}$; dots, $\theta=90^{\circ}$. Error bars indicate statistical errors only. The dotted lines indicate transmissions calculated from the model using constants obtained from transverse geometry fits.

were taken for a shorter time than the transverse geometry, and therefore have larger statistical uncertainties.

The salient features of the data can be accounted for by an extension of Eq. (2), assuming that the magnetic domains are needlelike, or laminar, with the thin dimension $\delta$. At each crystal orientation the average domain length traversed by a neutron will be increased by $1 /$ sine of the angle between the neutron momentum and the domain dimension $\delta$, as shown in Fig. 4. The neutron polarization transmission in the longitudinal geometry can then be represented by Eq. (2). However, we do not assume that the domains are magnetized parallel to the $c$ crystalline axis. Instead, we substitute in Eq. (2) the factor $\theta+\phi$, where $\phi$ is the angle (to be determined) between the domain magnetization axis and the $c$ crystalline axis. We assume $D_{\text {random }}^{\prime}$ is an orientation independent depolarization parameter and $D_{\text {bulk }}^{\prime}$ is a function of orientation $\theta$ through the $\delta /|\sin (\theta+\phi)|$ term. In the transverse geometry the rotation of the neutron polarization produces

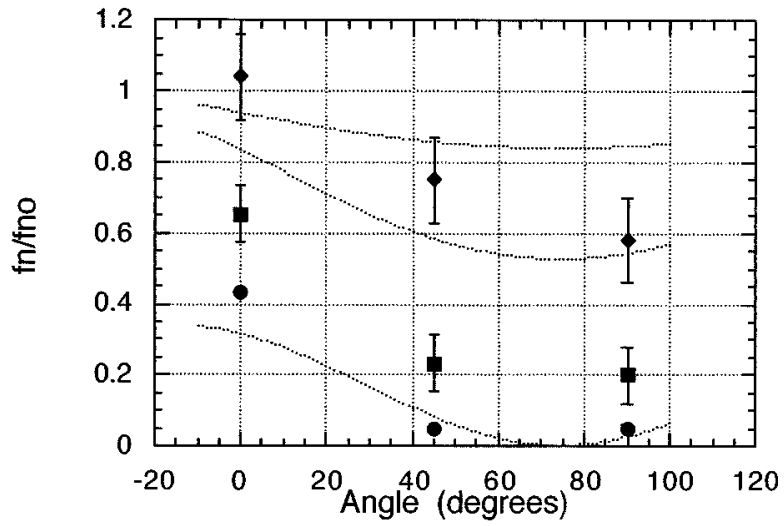

FIG. 3. The measured neutron polarization transmission $f_{n} / f_{n o}$ as a function of the crystal orientation at different energies for the transverse geometry. The $16 \mathrm{eV}$ (avg) data are the average of data from neutron energies of 14, 16 , and $18 \mathrm{eV}$. The dotted lines indicate $f_{n} / f_{n o}$ calculated from the model.

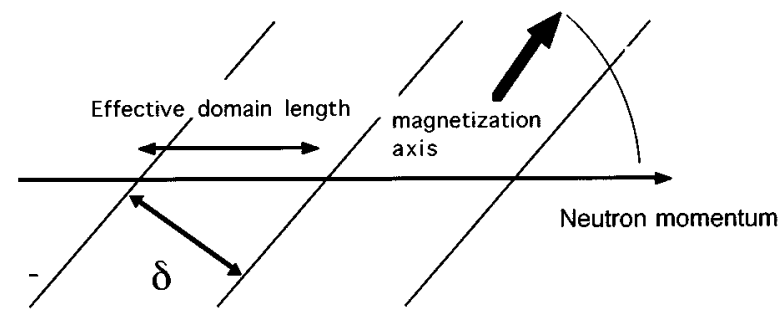

FIG. 4. The measured neutron polarization transmission $f_{n} / f_{n o}$ as a function of the crystal orientation for different energies in the longitudinal geometry. Dots, $E_{n}=1.7 \mathrm{eV}$; squares, $E_{n}=16 \mathrm{eV}$ (averaged); diamonds, $E_{n}=59 \mathrm{eV}$. The dotted lines indicate transmissions calculated from the model using constants from transverse geometry fits.

$$
\begin{aligned}
f_{n} / f_{n 0}= & {\left[\sin ^{2}(\theta+\phi)+\cos ^{2}(\theta+\phi) \exp \left(-2 D_{\text {bulk }}^{\prime} t\right)\right] } \\
& \times \exp \left(-2 D_{\text {random }}^{\prime} t\right) .
\end{aligned}
$$

The transverse $f_{n} / f_{n o}$ at each energy was fitted to Eq. (4) using a nonlinear fitting procedure. To reduce errors the 14 , 16 , and $18 \mathrm{eV}$ data were averaged together to one set before fitting. The 1.7 and $2.7 \mathrm{eV}$ data are insensitive to the value of $-2 D_{\text {bulk }}^{\prime} t$. Therefore this term was not included in those fits. The results of the fits are shown in Table I and in Fig. 5.

The weighted average value of the offset angle $\phi$ is $15.5^{\circ} \pm 0.8^{\circ}$. The other two parameters were fit as functions of $1 / E_{n}$, as expected from Eqs. (1) and (3), to obtain $D_{\text {random }}^{\prime}=0.58 / E_{n}(\mathrm{eV} / \mathrm{cm})$ and $D_{\text {bulk }}^{\prime}=2.8 / E_{n}(\mathrm{eV} / \mathrm{cm})$. From Eq. (3) and the average cylinder thickness $t=1.57 \mathrm{~cm}$, we find the domains have an average width of $\delta=59 \pm 6 \mu \mathrm{m}$. Because the $59 \mathrm{eV}$ neutrons are completely depolarized at some angles, the domain length in the long dimension is presumed to be greater than the $880 \mu \mathrm{m}$ limit discussed above.

Because the longitudinal polarization transfer data were more sparse, we did not fit the results shown in Fig. 3, but compare them instead to the $f_{n} / f_{n o}$ values calculated from Eq. (2) using values of the depolarization constants determined from the transverse data. Although the quantitative agreement is poor, it is seen that, at sufficiently high neutron energies, $f_{n} / f_{n o}$ is nonzero for all angles.

In summary we have presented measurements of the polarization transfer of epithermal neutrons through a thick single crystal of ferromagnetic holmium. The dependence of the polarization transfer on neutron energy and the orientation of the crystal is qualitatively explained by a model of

TABLE I. Depolarization parameters determined at selected neutron energies $E_{n}$.

\begin{tabular}{lccc}
\hline \hline$E_{n}(\mathrm{eV})$ & $-2 D_{\text {random }}^{\prime} t$ & $-2 D_{\text {bulk }}^{\prime} t$ & $\phi(\mathrm{deg})$ \\
\hline 1.7 & $-0.944 \pm 0.08$ & Not fit & $11.0 \pm 3.4$ \\
2.7 & $-0.816 \pm 0.06$ & Not fit & $14.9 \pm 2.6$ \\
16 (avg) & $-0.107 \pm 0.04$ & $-0.49 \pm 0.06$ & $12.5 \pm 1.2$ \\
36 & $-0.242 \pm 0.11$ & $-0.14 \pm 0.06$ & $18.6 \pm 2.2$ \\
59 & $-0.045 \pm 0.05$ & $-0.26 \pm 0.04$ & $19.0 \pm 1.4$ \\
\hline \hline
\end{tabular}



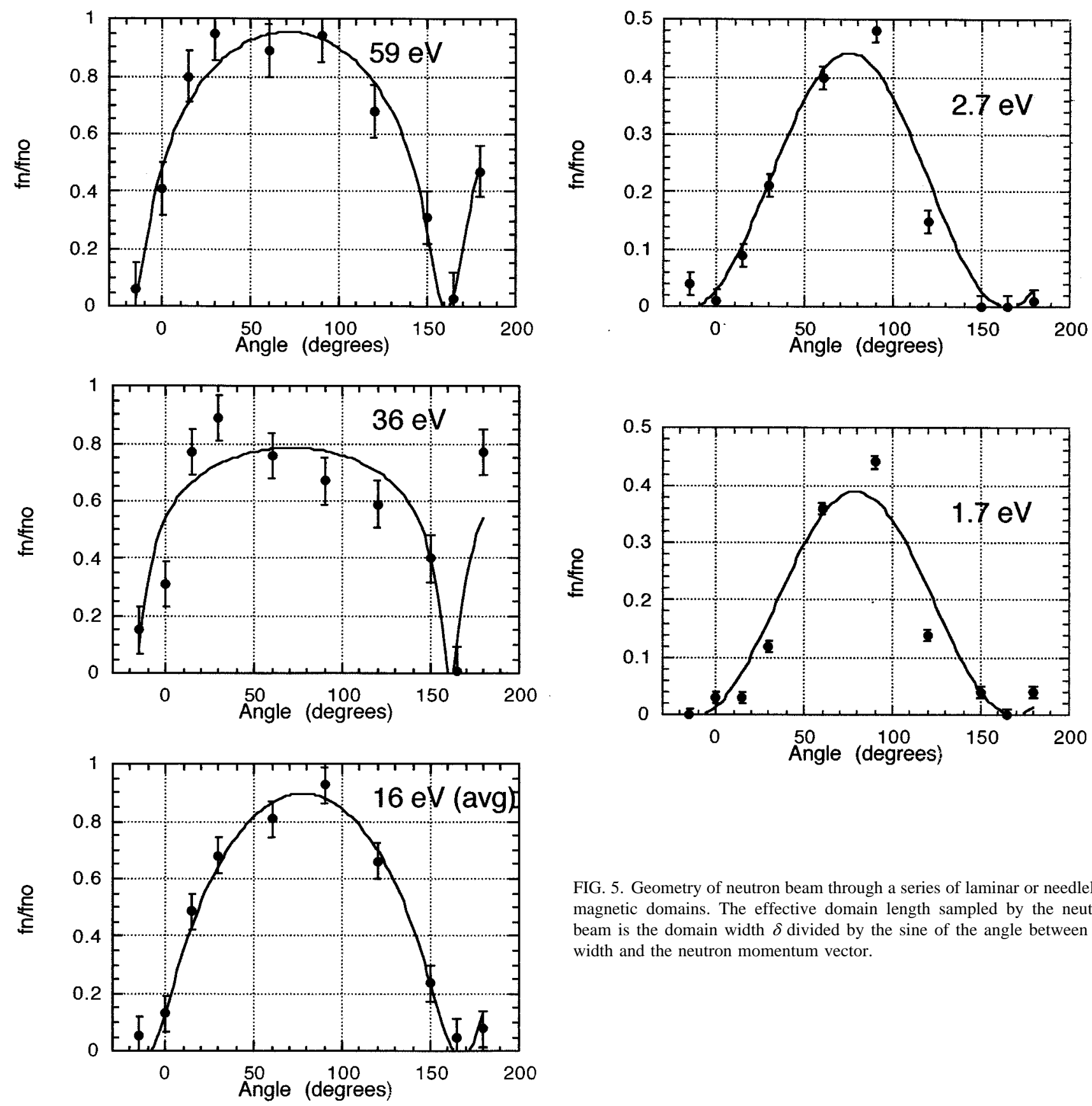

FIG. 5. Geometry of neutron beam through a series of laminar or needlelike magnetic domains. The effective domain length sampled by the neutron beam is the domain width $\delta$ divided by the sine of the angle between the width and the neutron momentum vector.

laminar or needlelike magnetic domains having a minimum dimension slightly offset from the $c$ crystalline axis. The method can be extended to other magnetic systems having large domains or large magnetizations.

\section{ACKNOWLEDGMENTS}

The authors gratefully acknowledge the helpful comments of A. Masaike and K. Okumura. This research was supported by Grant No. NK 1300 from the International Science Foundation by the Russian Government, and by the U.S. Department of Energy, Office of High Energy and Nuclear Physics, under Contract Nos. DE-AC05-76ER01067 and DE-FG05-88-ER40441.

${ }^{1}$ W. G. Williams, Polarized Neutrons (Clarendon, Oxford, 1988).

${ }^{2}$ D. L. Strandburg, S. Legvold, and F. H. Spedding, Phys. Rev. 127, 2046 (1962).

${ }^{3}$ O. Halpern and T. Holstein, Phys. Rev. 59, 960 (1941).

${ }^{4}$ H. Postma, H. Marshak, V. L. Sailor, F. J. Shore, and C. A. Reynolds, Phys. Rev. 126, 979 (1962)

${ }^{5}$ D. G. Haase, J. D. Bowman, P. P. J. Delheij, C. M. Frankle, C. R. Gould, J. N. Knudson, J. E. Koster, G. E. Mitchell, S. Penttila, H. Postma, N. R. Roberson, S. J. Seestrom, S. H. Yoo, and V. W. Yuan, Phys. Rev. B 46, 11290 (1992).

${ }^{6}$ W. H. Kraan and M. Th. Rekveldt, J. Magn. Magn. Mater. 5, 247 (1977). ${ }^{7}$ V. P. Alfimenkov, Yu. D. Mareev, V. V. Novitsky, L. B. Pikelner, V. R. Skoy, C. R. Gould, D. G. Haase, and N. R. Roberson, Nucl. Instrum. Methods Phys. Res. A 352, 592 (1995).

${ }^{8}$ V. P. Alfimenkov, S. B. Borzakov, J. Wierzbicki, A. I. Ivanenko, Yu. D. Mareev, O. N. Ovchinnikov, L. B. Pikelner, and E. I. Sharapov, Nucl. Phys. A 376, 229 (1982). 